\title{
Effect of Dry Cupping Therapy on Pain and Functional Disability in Persistent Non-Specific Low Back Pain: A Randomized Controlled Clinical Trial
}

\author{
Marianna de Melo Salemi ${ }^{1, *}$, Vanessa Maria da Silva Alves Gomes ${ }^{1}$, Laylla Marjorye Rebouças Bezerra', \\ Thania Maion de Souza Melo', Geisa Guimarães de Alencar', Iracema Hermes Pires de Mélo Montenegro², \\ Alessandra Paula de Melo Calado ${ }^{3}$, Eduardo José Nepomuceno Montenegro', Gisela Rocha de Siqueira ${ }^{1}$
}

${ }^{1}$ Department of Physiotherapy, Federal University of Pernambuco, Recife, Brazil

${ }^{2}$ Department of Nutrition, University of Pernambuco, Petrolina, Brazil

${ }^{3}$ Department of Surgery, Federal University of Pernambuco, Recife, Brazil

Received January 27, 2021

Revised May 10, 2021

Accepted September 7, 2021

Correspondence to Marianna de Melo Salemi Department of Physiotherapy, Federal University of Pernambuco, Recife, PE, Brazil

E-mail mariannasalemifisio@gmail.com
Background: Cupping therapy is used to treat musculoskeletal conditions, including low back pain.

Objectives: The study assessed the effects of dry cupping on pain and functional disability from persistent nonspecific low back pain.

Methods: This was a randomized controlled trial, where participants were allocated to a cupping therapy $(n=19)$ or sham $(n=18)$ group, for five 10-minute sessions of cupping therapy, twice a week, to stimulate the acupoints related to low back pain (GV4, BL23, BL24, BL25, and BL30, BL40 and BL58) and emotional aspects (HT3 and ST36). All participants were assessed at baseline, post-treatment and follow up (a finalization period of four weeks) using a visual analogue scale (VAS) and the Oswestry Disability Index (ODI). Groups were compared using the analysis of covariance (ANCOVA) and the effect size was calculated using Cohen's $d$.

Results: The cupping therapy group presented a lower mean VAS when compared to the sham, at post-treatment (mean difference: -2.36 ; standard error [SE]: $0.58 ; p<0.001$; "large" effect size: -0.94 ) and follow up (mean difference: -1.71 ; SE: $0.81 ; p<0.042$; 'large' effect size: -0.83$)$. The cupping therapy group presented a lower mean ODI when compared to the sham post-treatment (mean difference: -4.68 ; SE: 1.85; $p$ : 0.017; 'large' effect size: -0.87 ), although in follow-up, there was no difference between the groups (mean difference: 4.16; SE: 2.97; $p$ : 0.17; "medium" effect size: -0.70 ).

Conclusion: Dry cupping was more effective in improving pain and functional disability in people with persistent nonspecific low back pain when compared to the sham.

Keywords: Low back pain, Disabled persons, Cupping, Sham treatment

\section{INTRODUCTION}

Low back pain is a condition that causes a significant impact on people's functionality and quality of life, and is classified as persistent, when symptoms last more than three months [1-3] and nonspecific, when the cause of the pain is not determined [4].

Nonspecific low back pain is the most frequent condition amongst the causes of low back pain [5] and, for its physiotherapeutic treatment, therapeutic exercises and manual therapy are generally recommended [6]. Complementary therapies, such as cupping therapy, are resources that may be used to relieve low back pain and over recent years, have presented satisfactory results in terms of improving pain and functionality [7-9].

Dry cupping and wet cupping are the two main types of application, in which suction is applied to the skin at a specific acupuncture point (acupoint) or region. The difference between the two types of cupping therapy is due to the fact that wet cupping is performed through a small skin incision through which blood is drawn [10]. Both types have similar effects in terms of relieving low back pain [11].

However, dry cupping is a non-invasive intervention and is considered safer, easier to apply and presents fewer adverse 
effects when compared to wet cupping [12].

Treatment with dry cupping may present some variations regarding the suction method. Suction may be created by means of generating a vacuum with fire, and is called fire cupping, with the use of an aditional substance known as moxa [8]. However, this practice presents certain disadvantages since it is considered unsafe due to the possibility of causing lesions and burns to the skin of the recipients. In contrast, suction may also be achieved by means of a manual instrument, a hand pump, which in the literature, is considered to be a safe and easy manner to obtain suction in the cup $[8,13]$.

Thus, the use of dry cupping therapy may be used as a treatment tool for low back pain [11]. However, as yet, studies on the effectiveness of this technique are scarce and in many studies there is still an incomplete description of the method and the intervention $[13,14]$.

Furthermore, there is no consensus in the literature regarding a dry cupping protocol, regarding treatment time (studies vary between 8 and 20 minutes) and number of sessions (varying from one to eight visits) [15-18] necessary for pain relief and improved functionality.

In relation to the acupoints, points BL23, BL24 and BL25 are the most commonly used, either individually or together to relieve low back pain through cupping therapy [18-20]. Additional points of the governing vessel meridian and the bladder (GV4, BL30, BL40 and BL58) are acupoints which, although not used in low back pain surveys, are nonetheless related to low back pain and strengthening the lower back region [9,21-23], and should be included in treatment for a more comprehensive approach to low back pain.

The literature also suggests that interventions to control low back pain should associate conducts that act on the control of emotional and psychosocial issues, and which are related to the genesis and chronification of low back pain $[24,25]$ and the determinants of disability [26-28]. Thus, for the treatment of low back pain, the HT3 and ST36 points are related to the heart and stomach meridians, since they act on depression, insomnia and anxiety, stabilizing the mind and emotions, and also influence the improvement of painful symptoms [29].

No studies were discovered using points related to these psychosocial factors, which are considered by the literature to be predictive factors of disability related to low back pain [3032].

It may therefore be hypothesized that performing cupping therapy combined with stimulating the above mentioned acupoints (BL23, BL24 and BL25) [16,19] with other points associated with the aspect of low back pain (GV4, BL30, BL40 and BL58) and points related to the emotional condition (HT3, ST36) [9] may have a greater effect on improving pain and functionality.
Based on the above, the aim of the present study is to assess the effects of cupping therapy on pain and functional disability in individuals with persistent nonspecific low back pain. In addition, it will also assess the impact of cupping therapy on physical and psychosocial factors and the number of days per week of low back pain.

\section{MATERIALS AND METHODS}

\section{Type of study}

This was a randomized controlled clinical trial. The study was conducted according to CONSORT [33] registered on the Brazilian Registry of Clinical Trials (RBR-3s7g4t) and approved by the Ethics and Research Committee at the Universidade Federal de Pernambuco (Protocol number: 3.492.806). All participants signed the informed consent form.

\section{Participants}

Adults with nonspecific low back pain (for more than three months) aged between 18 and 59 years were included in the study. They were excluded if: they were taking anticoagulants, pregnant, women in the puerperium period, presented with anemia, presented red flags of low back pain [34], presented with systemic diseases, fibromyalgia, herniated disk in the lumbar region, symptomatic irradiated pain, previous spine surgery, altered skin integrity or had previously undergone treatment with cupping therapy.

\section{Randomization, allocation and blinding}

Participants were randomized into two groups (cupping therapy and sham) in blocks of 10, using the program www. randomization.com, thereby having allocation confidentiality. The study was blinded to evaluators, participants and statisticians to compare the effect of cupping therapy and the sham group.

In order to ensure blinding to the random distribution process, the allocation of each patient to one of the study groups was coded through computer systems by a researcher uninvolved in the study, and the treatments that they would receive were delivered in sealed opaque envelopes. Participants remained uninformed regarding the group to which they had been allocated throughout the study. In addition, participants received no prior knowledge regarding the technique, and the groups were collected in two different environments, thus avoiding communication among themselves about the intervention, in order to control blinding.

\section{Intervention}

Participants in both groups received five sessions, which took place twice a week, with a total treatment time of 20 
minutes, including the application of the suction cups in the anterior region (10 minutes) and then in the posterior region (10 minutes) of the body. The cups remained on the acupoints during a period of 10 minutes $[19,20]$. Seventeen reusable acrylic cups (Dong Yang) of medium diameter $(3.5 \mathrm{~cm})$, were used for each individual, at each session. Before beginning the session, if any signs of dehydration were identified by the therapist, coconut oil was applied to the region in order to hydrate the skin [35].

In order to locate the points, an individual measurement was used for each patient, known as tsun (cun). Tsun, or cun, is the distance or fraction fixed between two determined references, bone or morphological, used to locate the points. It is the most appropriate measurement since it provides proportionate measures for each person [36].

During data collection, the only additional procedures patients received were cupping therapy or sham, however they also performed routine drug treatment according to medical prescription.

The professionals who identified the points and performed the interventions had been graduated with experience in treating low back pain for over six years and with more than three years of experience in the area of traditional Chinese medicine.

\section{1) The cupping therapy group}

The cups, using the dry cupping technique, were initially applied through two suctions by the hand pump, "moderate" suction, which generate a negative pressure of approximately 300 millibar [37]. For participants who reported considerable discomfort, the pressure was slightly minimized according to the individual sensation. Each cup was positioned so that the acupoint was in the center of the cup. The vacuum was created only with the use of a hand pump and no other instrument or substance.

The intervention protocol for the cupping therapy group was developed based on a previous study that suggested specific acupuncture points, BL23 (Shenshu), BL24 (Qihaishu) and BL25 (Dachangshu) for the treatment of low back pain [21]. Additional points were included in this protocol related to emotional factors, HT3 (Shaohai) and ST36 (Zusanli) [29] and related to low back pain, GV4 (Mingmen), BL30 (Baihuanshu), BL40 (Weizhong) and BL58 (Feiyang) [21-23].

To apply the cups, the participants were initially placed in the supine position and four cups were applied on the following points: HT3 (Shaohai) and ST36 (Zusanli), bilaterally, for approximately ten minutes.

Following this, the participants moved into the prone position, with 13 cups placed bilaterally on the following points: GV4 (Mingmen), BL23 (Shenshu), BL24 (Qihaishu), BL25 (Dachangshu), BL30 (Baihuanshu), B40 (Weizhong) and BL58 (Feiyang). The cups remained in place for a further ten minutes (Fig. 1).

\section{2) The sham group}

The cups were positioned following the same protocol and acupoints as the cupping therapy group. However, the wall of these cups had a small hole (made with a heated $1.9 \mathrm{~mm}$ crochet hook), which prevented the applied suction from being maintained (Fig. 2).

In order for the cups to remain attached to the skin in each region, a transparent double-sided adhesive tape was used along the edge of the cup. With regard to ethical issues, so that the sham group could receive an effective treatment for low back pain, once the study had been completed, all participants received treatment with real cupping therapy as recorded with the ethics committee. Those patients who did not present improvement with cupping treatment were offered traditional physiotherapy.

\section{Adverse effects and follow-up control}

After applying the cupping therapy, an alteration is expected at certain points in the skin pigmentation, plus a slight increase in sensitivity/pain (VAS $<4$ ) in the region of where the cup was applied [38]. Therapy would be interrupted if participants presented either blisters and/or skin eruptions at the points receiving the applications.

\section{Outcomes}

The primary outcomes were intensity of pain and functional disability. Pain was measured using the visual analogue scale (VAS) [39]. Functional disability was measured using the Oswestry Disability Index (ODI) questionnaire [40]. The secondary outcomes were the physical and psychosocial factors for people with low back pain through the questionnaire Start Back Screening Tool (SBST) [41], and a weekly pain diary (registering the number of days per week that the participant reported events of pain).

The variables VAS, ODI and SBST were assessed at three points in time, first at the initial assessment (baseline), then after five session (post-treatment) and at follow up (4 weeks post-treatment). The variable number of days per week of pain was assessed at baseline moments, after one week of treatment (two session), after two weeks (four session) of treatment and post-treatment (after five session).

\section{Sample size calculation}

The sample was calculated based on a pilot study $(n=30$; 15 per group) conducted by the researchers, considering the primary outcomes of this study. For the VAS, the following values were considered: cupping therapygroup (mean: 2.25; standard deviation: 1.71 ) and sham group (mean: 

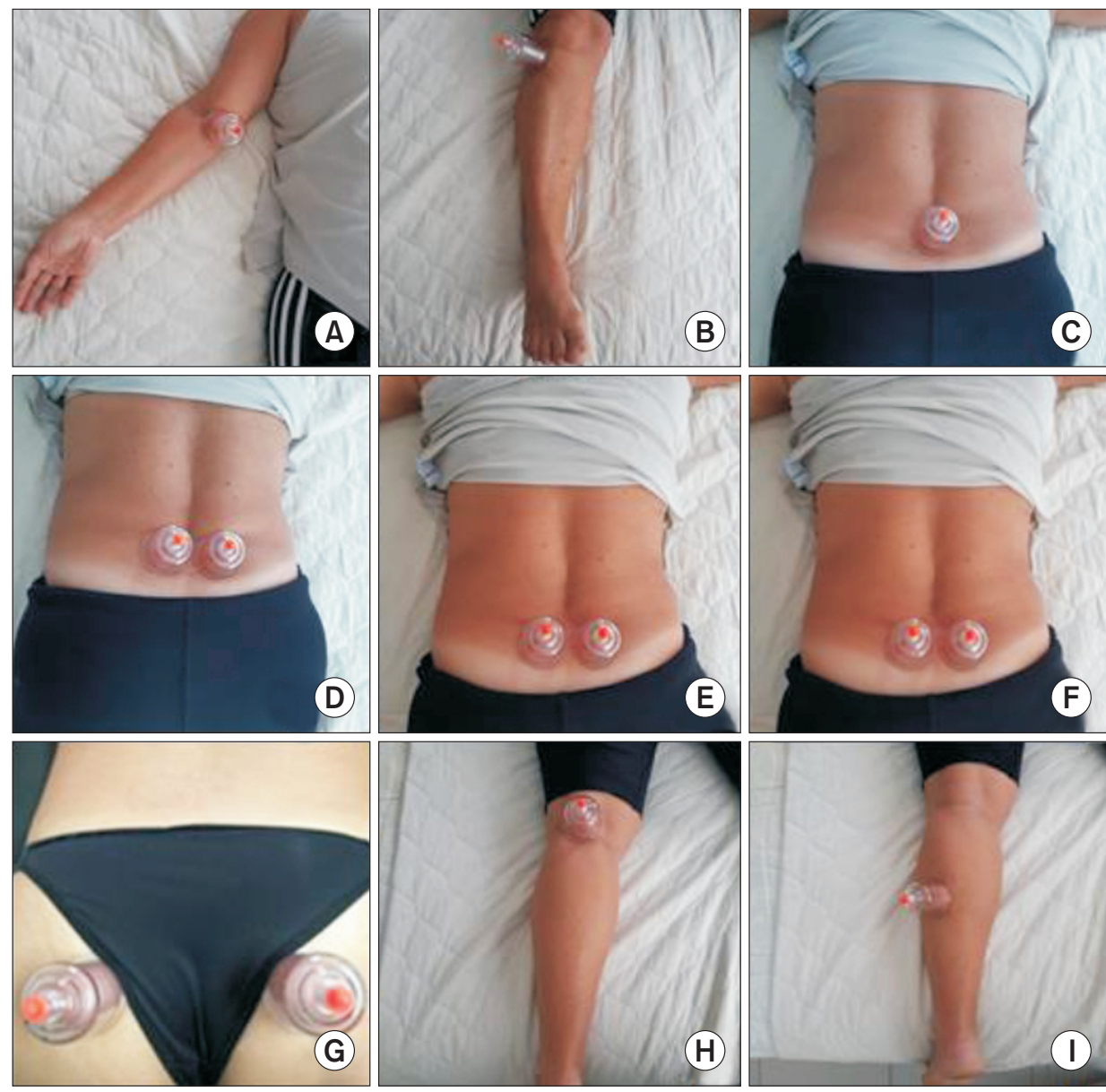

Fig. 1. Acupoints for low back pain. (A) HT3 (Shaohai), located on the ulnar surface of the skin fold, in the anterior region of the medial epicondyle over the tendon of the biceps brachii, at the medial end of the flexion fold; (B) ST36 (Zusanli), located on the antero-lateral side of the leg at the anterior margin of the tibia between the anterior tibial and common extensor muscles of the fingers; (C) GV4 (Mingmen), located in the depression below the spinous L2 process; (D) BL23 (Shenshu), in the lumbar region, below the spinous process of L2, 1.5 lateral cun; (E) B2L4 (Qihaishu), located in the lumbar region, below the spinous process of the third lumbar vertebra, 1.5 cun lateral to the posterior midline of L3; (F) BL25 (Dachangshu), in the lumbar region, below the spinous process of L4, 1.5 lateral cun; (G) BL30 (Baihuanshu), in the sacrum, at the level of the fourth posterior sacral foramen, 1.5 cun lateral to the median sacral crest; $(\mathrm{H}) \mathrm{BL} 40$ (Weizhong), located in the midpoint of the popliteal fold and (I) BL58 (Feiyang), located in the lateral region in the belly of the gastrocnemius muscle.

4.59; standard deviation: 2.18 ) and effect size: 1.179. For the ODI, the following values: suction cup group (mean: 11.44; standard deviation: 6.68) and sham group (mean: 18.12; standard deviation: 8.93 ) and effect size: 0.847 . Thus, considering $80 \%$ of power and $5 \%$ of alpha, the minimum sample was 17 participants per group, considering the ODI outcome that presented the highest estimate of sample size. Taking into account possible eventual losses, a sample of 19 participants per group was used $(n=38)$.

\section{Statistical analysis}

The statistical analysis was performed using SPSS 20.0. The Shapiro-Wilk test was used to analyze the normality of data. The characterization of the sample and the values of the baseline outcome variables were presented in absolute and percentage numbers and in mean and standard deviations. The comparability of variables between groups at baseline was ensured using the t-tests for independent samples for continuous data and the Pearson's $\chi^{2}$ tests, $\chi^{2}$ with continuity correction or the Fisher's $\chi^{2}$ tests, when appropriate, for categorical data.

For the variables VAS, ODI and STBS, univariate analyzes of covariance (ANCOVA) were performed, which modeled each post-treatment and follow-up result according to the treatment group - suction cup or sham (classified factor), and their respective baseline values (linear covariate). The estimated data from the post-treatment and follow-up results of ANCOVA were presented as mean and standard error. In addition, the mean of the difference between the groups, the standard error, the $95 \%$ confidence interval and the $p$-value 
were presented.

The effect size of the cupping therapy compared to the sham was calculated using the estimated mean and standard error of each group for the variables VAS, ODI and SBST and interpreted according to Cohen's criterion (small $\leq 0.2$ to 0.49 ; medium: 0.5 to 0.79 ; large $\geq 0.8$ ) [42].

For the outcome number of days per week of pain, repeated measures ANOVA and the Bonfferoni post-hoc test were used, considering four periods of assessment: baseline, after

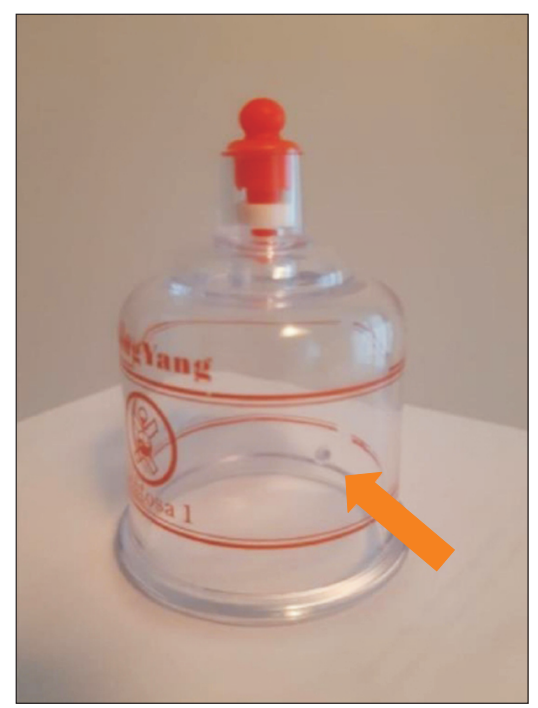

Fig. 2. Cup for the sham group. the first week of treatment, after the second week of treatment and post-treatment in each group. For the comparison between groups at each moment, the $t$ test for independent samples was performed.

To analyze whether the minimal important difference (MID) for the variable (VAS and ODI) was achieved in each group, the mean difference in the scores of each scale was obtained at baseline, post-treatment and follow up. The MID was analyzed in each treatment group (cupping therapy and sham) in relation to VAS (for which a MID was considered of 2 points on the scale) and ODI (for which a MID was considered of 10 points in the questionnaire) [43]. For this analysis, repeated measures ANOVA and the Bonferroni posthoc test were used. The absolute number and percentage of individuals who reached the MID at each point of assessment was also obtained.

There was no analysis of the intention to treat primary or secondary outcomes because there was no significant loss of individuals throughout the study.

\section{RESULTS}

\section{Participants}

Thirty-eight participants were randomized for the study (Fig. 3, CONSORT flowchart). There was only one participant loss in the sham group, so that 37 individuals completed all stages of the study. The characteristics of the participants who completed the study were similar between groups at baseline

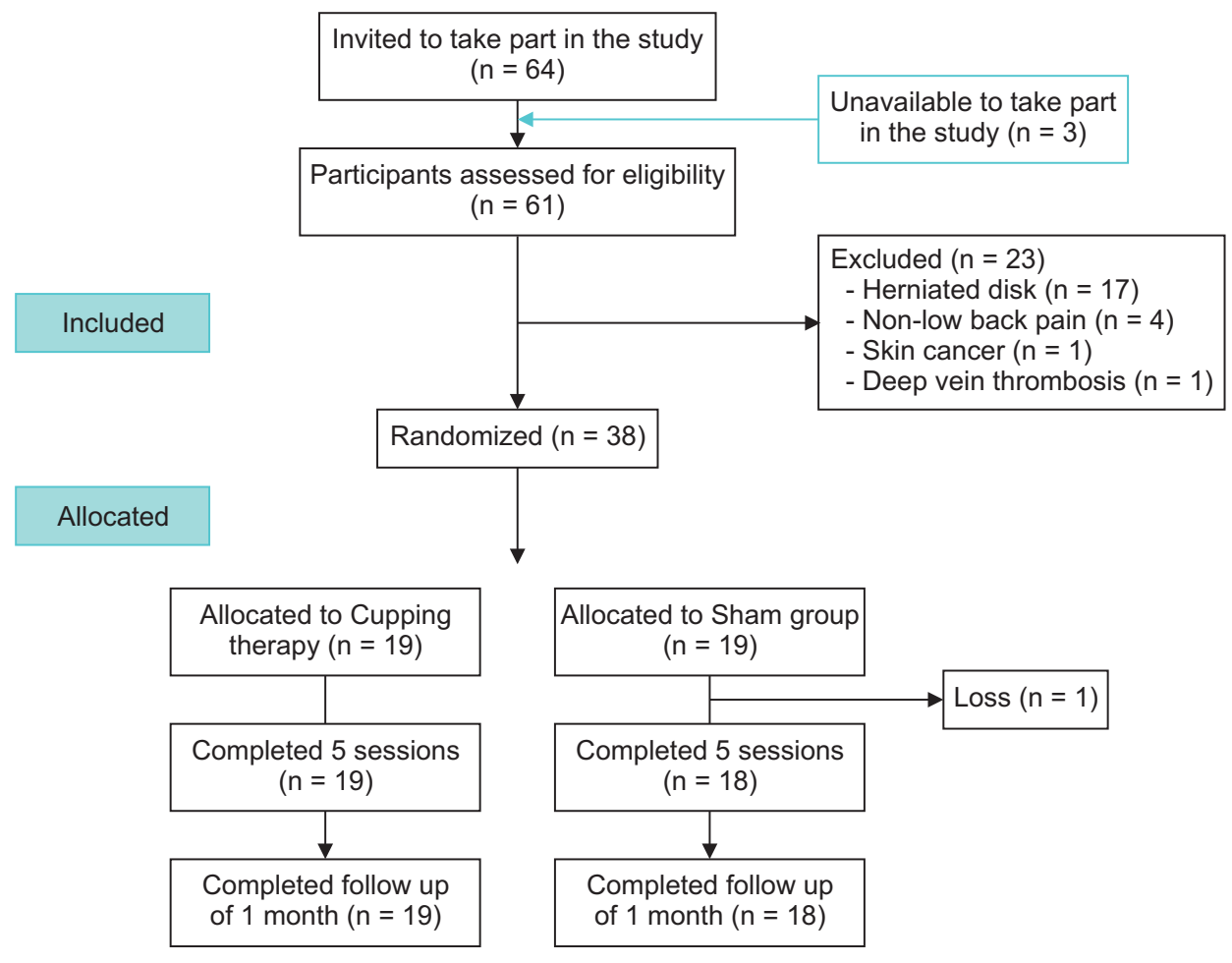

Fig. 3. Flowchart for selecting and monitoring participants. 
( $p$-values $>0.05)$ and are presented in Table 1.

\section{Primary outcomes}

The cupping therapy group presented a lower VAS at posttreatment (mean difference: 2.36; standard error: 0.58; 95\% CI: -3.55 to -1.17 ; $p$ : $<0.001$; 'large' effect size: -0.94 ) and follow up (mean difference: -1.71 ; standard error: 0.81; 95\% CI: -3.37 to -0.06 ; $p:<0.042$; 'large' effect size: 0.83 ) when compared to the sham group (Table 2).

With regards to ODI, the cupping therapy group presented a lower post-treatment score, (mean difference: -4.68 ; standard error: 1.85 ; $95 \%$ CI: -8.45 to $-0.90 ; p$ : $<0.017$; 'large' effect size: -0.87$)$ when compared to the sham group. At follow up, there were no differences between the groups (mean difference: -4.16 ; standard error: $2.97 ; 95 \%$ CI: -10.20 to 1.87 ; $p:<0.001$; 'medium' effect size: -0.70 ) (Table 2).

\section{Minimal Important Difference (MID) for ODI and VAS}

The cupping therapy group demonstrated a reduction in the pain score (VAS) between the post-treatment and baseline periods (mean difference -2.47 , standard error: 0.50, $p:<$ 0.001 ), and between the follow-up and baseline periods (mean difference -2.78 , standard error: $0.55, p:<0.001$ ). There was no difference between the follow-up and post-treatment periods (mean difference 0.31 , standard error: $0.36, p: 1,000$ ). In the sham group, there was no significant reduction in pain intensity between the moments of assessment.

In the percentage analysis per individual who achieved a MID, $68.42 \%$ of the participants in the cupping therapy group reached a MID for the VAS between the post-treatment and baseline periods (against 22\% in the sham group), $63.15 \%$ between the follow-up and baseline periods (against $50 \%$ in the sham group) and $26.31 \%$ between the follow-up and posttreatment periods (against $27.77 \%$ in the sham group).

In relation to the ODI, there was an improvement in the cupping therapy group in functional disability at the posttreatment period compared to the baseline (mean difference -6.52 , standard error: $1.45, p:<0.001)$ and between the followup and baseline periods (mean difference -8.63 standard error: $1.97, p:<0.001)$. No difference was observed between the follow-up and post-treatment periods (mean difference -2.10 , standard error: $1.00, p: 0.154$ ). In the sham group, there was no significant reduction in the ODI score between the assessment periods.

In the percentage analysis per individual who achieved a MID, 36.85\% of the participants in the cupping therapy group achieved a MID for the ODI between the post-treatment and baseline periods (against 16.66\% in the sham group), 36.85\% between the follow-up and baseline periods (against 38.85\%

Table 1. Baseline characteristics of the cupping therapy and sham groups

\begin{tabular}{|c|c|c|c|}
\hline Variables & Cupping therapy $(n=19)$ & Sham $(n=18)$ & $p$-value \\
\hline Age (years), mean (SD) & $26.68(7.64)$ & $23(2.47)$ & $0.060^{*}$ \\
\hline Sex, n $(\%)$ & & & $0.420^{*}$ \\
\hline Male & $6(31.6)$ & $9(50)$ & \\
\hline Female & $13(68.4)$ & $9(50)$ & \\
\hline Height (m), mean (SD) & $1.68(0.11)$ & $1.70(0.10)$ & $0.654 *$ \\
\hline Weight (kg), mean (SD) & 69.68(12.27) & $68.61(15.21)$ & $0.814^{*}$ \\
\hline IPAQ, n $(\%)$ & & & $0.518^{\ddagger}$ \\
\hline Sedentary and insufficiently active & $3(15.8)$ & $1(5.6)$ & \\
\hline Active (A \& B) & $13(68.4)$ & $15(83.3)$ & \\
\hline Very active & $3(15.8)$ & $2(11.1)$ & \\
\hline Occupation, n (\%) & & & $0.232^{\S}$ \\
\hline Students & $13(68.4)$ & $16(88.9)$ & \\
\hline Paid work & $6(31.6)$ & $2(11.1)$ & \\
\hline VAS (score), mean (SD) & $4.68(1.97)$ & $5.39(2.15)$ & $0.305^{*}$ \\
\hline ODI (score), mean (SD) & $17.79(7.63)$ & $21.78(9.99)$ & $0.179 *$ \\
\hline STBS (score), mean (SD) & $3.71(1.10)$ & $4.18(1.51)$ & $0.30^{*}$ \\
\hline STBS, n $(\%)$ & & & $0.611^{+}$ \\
\hline Low risk & $10(52.6)$ & 7 (38.9) & \\
\hline Medium and high risk & $9(47.4)$ & $11(61.1)$ & \\
\hline Pain diary (score), mean (SD) & $4.90(2.05)$ & $4.83(1.69)$ & $0.922 *$ \\
\hline
\end{tabular}

VAS = Visual Analogue Scale; ODI = Oswestry Disability Index; IPAQ = International Physical Activity Questionnaire; STBS = Start Back Screening Tool; $p=$ value referring to the comparison between the groups.

${ }^{*}$ t-teste for independent samples; ${ }^{\dagger}$ chi-squared test with continuity correction; ${ }^{\ddagger}$ Pearson's chi-square test; ${ }^{\S}$ Fisher's chi-square test. 
Table 2. Analysis of the outcomes between the groups undergoing treatment

\begin{tabular}{|c|c|c|c|c|c|c|}
\hline $\begin{array}{c}\text { Variables } \\
\text { Mean estimate (SE) }\end{array}$ & $\begin{array}{l}\text { Cupping } \\
\text { therapy }(n=19)\end{array}$ & Sham $(n=18)$ & $\begin{array}{l}\text { Mean difference } \\
\text { cupping therapy/ } \\
\text { sham (SE) }\end{array}$ & $\mathrm{Cl} 95 \%$ & $\begin{array}{l}p \text {-value } \\
\text { (cupping } \\
\text { therapy/ } \\
\text { sham) }\end{array}$ & Cohen d (effect size) \\
\hline \multicolumn{7}{|l|}{ VAS } \\
\hline Post-treatment & $2.39(0.41)$ & $4.75(0.42)$ & $-2.36(0.59)$ & -3.55 to -1.17 & $<0.001^{*}$ & -0.94 (large) \\
\hline Follow up & $2.08(0.56)$ & $3.80(0.58)$ & $-1.72(0.81)$ & -3.37 to -0.07 & $0.042 *$ & -0.83 (large) \\
\hline \multicolumn{7}{|l|}{ ODI } \\
\hline Post-treatment & $12.43(1.28)$ & $17.11(1.31)$ & $-4.68(1.85)$ & -8.45 to -0.91 & $0.017^{+}$ & -0.87 (large) \\
\hline Follow up & $10.08(2.05)$ & $14.25(2.11)$ & $-4.17(2.97)$ & -10.21 to 1.88 & $0.17^{\dagger}$ & -0.70 (medium) \\
\hline \multicolumn{7}{|l|}{ STBS } \\
\hline Post-treatment & $1.44(0.34)$ & $2.48(0.34)$ & $-1.46(0.53)$ & -2.46 to -0.38 & $0.01^{\ddagger}$ & -0.89 (large) \\
\hline Follow up & $1.37(0.31)$ & $2.32(0.31)$ & $-1.05(0.49)$ & -2.05 to -0.05 & $0.04^{\ddagger}$ & -0.83 (large) \\
\hline
\end{tabular}

SE = Standard Error; VAS = Visual Analogue Scale; ODI = Oswestry Disability Index; STBS = Start Back Screening Tool.

*ANCOVA estimates considering Baseline VAS scores as covariates; ${ }^{\dagger}$ ANCOVA estimates considering Baseline ODI scores as covariates; ${ }^{\ddagger}$ ANCOVA estimates considering Baseline STBS scores as covariates.

in the sham group) and 5.2\% between the follow-up and posttreatment periods (against $22.22 \%$ in the sham group).

\section{Secondary outcomes}

With regard to the SBST scores, the cupping therapy group demonstrated a lower rate both in the post-treatment (mean difference: -1.46 ; SE: 0.53 ; 95\% CI: -2.54 to -0.38 ; $p$ : $<0.01$, 'large' effect size: -0.89 ) and follow-up periods (mean difference: -1.04 ; SE: 0.49 ; 95\% CI: -2.04 to $-0.04 ; p<0.04$; 'large' effect size: -0.83 ) when compared to the sham group.

In the weekly pain diary, both groups demonstrated a reduction in the number of days of reported pain during the weeks of the study (repeated measures ANOVA, $p<0.01$ ). In the cupping therapy group, this reduction in the number of days of reported pain occurred between the baseline and the first week (post hoc: 0.001), and the second week (post hoc: < 0.0001 ) and post-treatment (post hoc: $<0.0001$ ). In the sham group, the difference occurred between the baseline and the second week (post hoc: < 0.0001) and post-treatment (post hoc: 0.02 ). When comparing the groups, it was observed that the reduction in the number of days was greater in the cupping therapy group after the first week $(p<0.01)$ and in the post-treatment $(p<0.02)$ (Fig. 4).

\section{Adverse effects}

None of the participants demonstrated any moderate or severe signs or symptoms that would have made it necessary to leave the study. Just one adverse event was registered involving a slight change in skin pigmentation in most of the participants $(n=19)$, but which disappeared within four days.

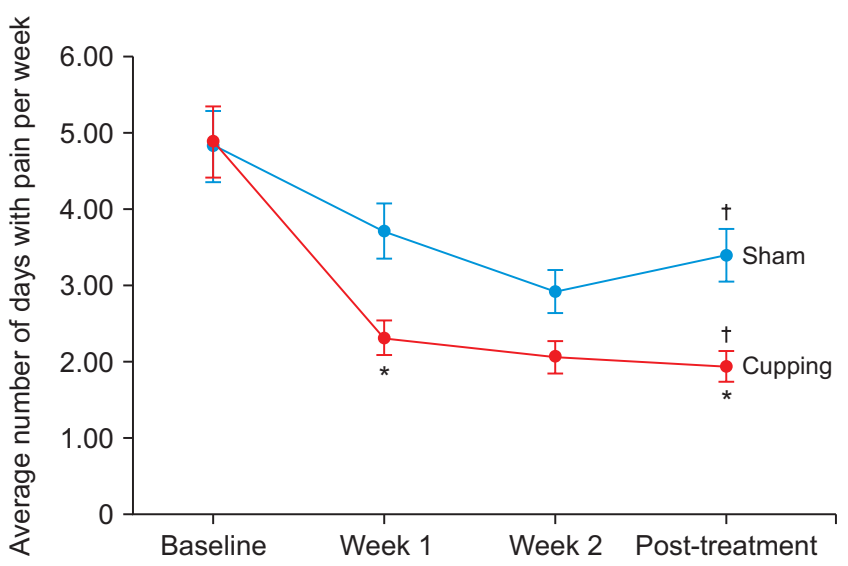

Fig. 4. Diary of days with low back pain. ${ }^{*} p<0,05$; t-test for independent samples (comparison between groups, at each assessment period). ${ }^{\dagger} p<0,05$; repeated measures ANOVA (comparing Baseline, Week 1, Week 2, and Post-treatment in each treatment group).

\section{DISCUSSION}

Dry cupping therapy is a treatment option for low back pain which provides satisfactory results in several types of low back pain $[15,16]$. According to the Eastern perspective, through stimuli on acupoints, it is possible to regulate the energy function, and consequently, the improvement of symptoms $[9,16,44-46]$.

From the viewpoint of Western medicine, the main theories and hypotheses related to the physiological effects of pain reduction would be the gate control theory of pain, the control of diffuse harmful inhibitors and theory of the reflection zone, in which pain relief is achieved through the inhibition of receptors conducting pain stimuli $[8,11,13]$ 
Acupoints BL23, BL24 and BL25 are the exact points for treating nonspecific low back pain $[19,20,47]$. However, it is important to consider the multi-causality of this pain condition [48-50], including psychosocial factors, which are strongly related to the onset of pain $[51,52]$.

To the best of our knowledge, thus far, the present study is a pioneer in addressing dry cupping therapy, considering acupoints correlated with emotional factors (HT3 and ST36) associated with local (GV4, BL23, BL24, BL25 and BL30) and distal points (BL40 and BL58) related to low back pain $[9,53,54]$.

It was found in the present study that the cupping therapy group demonstrated a significant improvement in pain intensity when compared to the sham group, with a mean difference of $-2.36(0.58)$ points on the VAS scale after treatment and $-1.71(0.81)$ points at follow up. A study by Volpato et al. [15], which used acupoints BL23, BL24 and BL25 compared a cupping therapy group with a sham, also reported an improvement in pain intensity, with a difference of 3.78 points between the groups at the post-treatment period and 3.23 points after a week of follow up, measured using the Brief Pain Inventory (BPI) [55].

The results of the present study and of Volpato et al. [15] demonstrate that cupping therapy has both immediate and delayed effects on improving low back pain. In posttreatment, the present study presented a large effect size, similar to the study by Volpato et al. [15]. In relation to follow up, it is important to note that Volpato et al. [15] only reassessed the participants one week after a single cupping session, which prevented discovering whether the effects of a single session are sufficient to last beyond one week. However, according to the present study, five sessions achieved a large effect on improving pain, after four weeks of follow-up.

The combined treatment of cupping therapy with medications has also been investigated [16]. Teut et al. [16], associated the use of pulsatile dry cupping with analgesics amongst three groups: minimum pressure group (-70 mbar), the cupping therapy group ( -150 to -350 mbar) and a control group. Participants in the three groups used paracetamol throughout the study period and it was reported that both the minimum pressure and cupping therapy groups, after eight sessions demonstrated a significant improvement in pain intensity $(p<0.001)$ when compared to the control group. However, after 12 weeks of follow up, only the cupping therapy group demonstrated a significant difference when compared to the control.

Given the previous evidence, the negative pressure considered as moderate, used in the cupping therapy group [16] was more effective in promoting a more lasting effect on the intensity of pain when compared to the group that only used the medication or the one that used minimum pressure.
This result demonstrates the impact of negative dry cupping pressure on relieving low back pain and reinforces the results of the present study, which achieved a large effect size on both the post-treatment and follow-up pain relief without being associated to any medication, using a pressure of approximately $300 \mathrm{hPa}$.

Moreover, it should be noted that the present study did not use pulsatile cupping therapy, as Teut et al. [16] did, but used a hand pump to generate the negative pressure of the suction cup on the skin, the pressure of which was graded depending on each individual's sensitivity threshold, and was maintained until the end of the session. However, pain relief results were similar across both studies so that continuous or pulsating pressure may have similar effects on pain intensity.

Cupping therapy has also demonstrated effects on low back pain in different populations, as observed in the study by Akbarzadeh et al. [56], whose sample was composed of women in the puerperium period. This study reported an improvement in the low back pain of the experimental group (dry cupping over the BL23 point) compared to the control (without treatment), through the McGill Pain Questionnaire.

However, one factor that should be considered in relation to the use of dry cupping is the placebo effect of applying the suction cup to the skin, which was not controlled in the study by Teut et al. [16] nor in the study by Akbarzadeh et al. [56], unlike the present study. Thus, the results of Teut et al. [16] and Akbarzadeh et al. [56] may have been overestimated due to the lack of control over the placebo effect.

In addition to reducing pain, in the present study there was also a reduction in the days of reported low back pain during the weeks of the study, measured by the weekly pain diary. It was observed that in the cupping therapy group the difference was perceived from the first week, continuing on through the second and after treatment. In the sham group there was a difference after the second week which continued into the post-treatment period, but when comparing the groups, the cupping therapy group demonstrated a greater significant difference. Since no studies were found in the literature that had made this assessment, these results could not be compared with other similar studies.

It is expected that with an improvement in low back pain, individuals will gradually return to their functionality $[4,49]$. In the present study, the cupping therapy group presented less post-treatment disability when compared to the sham group, with a difference of -4.68 (1.85) on the ODI scale, with an effect size considered as large. In the follow up, the cupping therapy and sham group demonstrated no differences in relation to the ODI. Considering that most cases of nonspecific low back pain are self-limiting, this result suggests that cupping therapy may produce a faster improvement in low back pain and functionality, which is maintained after 
treatment has ceased [57]. For the group that did not receive treatment the improvement of pain and functionality should occur over a longer period of time, respecting the course of the nonspecific pain.

Functional disability related to low back pain has also been benefited by the dry cupping method in previous studies $[15,16]$. In the study by Teut et al. [16] a significant improvement in the score of the disability questionnaires used by the authors (Funktionsfragebogen Hannover Rücken) was achieved only in the cupping therapy group when compared to the control group, in the post-treatment period. In contrast, this improvement in the cupping therapy group was not maintained after 12 weeks of follow up. The treatment proposed by Volpato et al. [15] also improved the outcome of functional disability (assessed by the RolandMorris disability questionnaire), however this was somewhat late, one week after follow up.

The fact that the treatment in this study included more acupuncture points and other regions related to low back pain, as well as the inclusion of points related to psychosocial factors, may have contributed to this improvement both in an immediate manner when achieving a MID with the VAS and also at a later date one month after follow up.

Through this approach, also contemplating the points attributed to emotional factors, it may be observed that the participants in the cupping therapy group of the present study demonstrated a greater clinical improvement regarding the prognosis of low back pain according to the SBST scores, when compared to the sham group, both in the posttreatment and follow-up periods. These findings configure the relationship between pain and functional disability with the biopsychosocial factor, which is mediated by the level of self-efficacy and fear $[4,58]$.

Therefore, while cupping therapy is a resource that may be used to treat low back pain and improve functional disability, as observed in the present study, it is also known that this method, even though it is considered safe and easy to apply, may present some adverse effects $[8,12,59]$. The most common are anemia and panniculitis [38] and are more frequent in wet cupping therapy [60] In addition, the importance was also emphasized of the qualifications of the professional in avoiding major complications [29]. The present study performed all the necessary procedures in order to minimize adverse effects. The only event was the occurrence of a slight change in skin pigmentation in most participants, which disappeared within four days, an effect expected after the intervention [37].

\section{Limitations}

The results of this study should be interpreted taking into account certain limitations. Since the majority of study participants were young females, the significance of the study results were limited in respect of older individuals with low back pain. A larger sample size for the study is needed to cover a wider range of patients with non-specific low back pain. Another limiting factor refers to the outcome results of the pain diary in the follow-up period not being presented, since this could have generated a forgetfulness bias by being a retrospective fact when assessed by the participants.

\section{CONCLUSIONS}

Treatment with cupping therapy was more effective in improving pain and functional disability in people with persistent nonspecific low back pain when compared to the sham group. It also brought about an improvement in the number of days of reported low back pain throughout the treatment as well as the prognosis of patients with persistent nonspecific low back pain.

\section{AUTHORS' CONTRIBUTIONS}

MMS: Data collection, manuscript writing, discussion of results, comments on article and submission, VMSAG: Statistical analysis, discussion of results and comments on article, LMRB: Discussion of results and comments on article, TMSM: Discussion of results and comments on article, GGA: Manuscript writing, statistical analysis, discussion of results and comments on article, IHPMM: Discussion of results and comments on article, APMC: Discussion of results and comments on article, EJNM: Discussion of results and comments on article, GRS: Manuscript writing, statistical analysis, supervision, corrections, final revision, discussion of results and comments on article.

\section{CONFLICT OF INTEREST}

The authors declare no conflict of interest.

\section{ORCID}

Marianna de Melo Salemi, https://orcid.org/0000-0001-5804-6181

Vanessa Maria da Silva Alves Gomes, https://orcid.org/0000-0002-5421-6654

Laylla Marjorye Rebouças Bezerra, https://orcid.org/0000-0002-9225-7932

Thania Maion de Souza Melo, https://orcid.org/0000-0002-8687-7836

Geisa Guimarães de Alencar, https://orcid.org/0000-0002-1240-135X

Iracema Hermes Pires de Mélo Montenegro, 
https://orcid.org/0000-0003-2770-8060

Alessandra Paula de Melo Calado,

https://orcid.org/0000-0001-9181-0209

Eduardo José Nepomuceno Montenegro, https://orcid.org/0000-0001-9798-9190

Gisela Rocha de Siqueira, https://orcid.org/0000-0003-4520-1175

\section{REFERENCES}

1. Bernstein IA, Malik Q, Carville S, Ward S. Low back pain and sciatica: summary of NICE guidance. BMJ 2017;356:16748.

2. Chenot JF, Greitemann B, Kladny B, Petzke F, Pfingsten M, Schorr SG. Non-specific low back pain. Dtsch Arztebl Int 2017;1 14:883-90.

3. Qaseem A, Wilt TJ, McLean RM, Forciea MA, Denberg TD, Barry MJ, et al. Noninvasive treatments for acute, subacute, and chronic low back pain: a clinical practice guideline from the American College of Physicians. Ann Intern Med 2017;166:51430.

4. Vibe Fersum K, Smith A, Kvåle A, Skouen JS, O'Sullivan P. Cognitive functional therapy in patients with non-specific chronic low back pain-a randomized controlled trial 3-year follow-up. Eur J Pain 2019;23:1416-24.

5. Maher C, Underwood M, Buchbinder R. Non-specific low back pain. Lancet 2017;389:736-47.

6. Foster NE, Anema JR, Cherkin D, Chou R, Cohen SP, Gross DP, et al. Prevention and treatment of low back pain: evidence, challenges, and promising directions. Lancet 2018;391:2368-83.

7. Lauche R, Spitzer J, Schwahn B, Ostermann T, Bernardy K, Cramer $\mathrm{H}$, et al. Efficacy of cupping therapy in patients with the fibromyalgia syndrome-a randomised placebo controlled trial. Sci Rep 2016;6:37316.

8. Aboushanab TS, AlSanad S. Cupping therapy: an overview from a modern medicine perspective. J Acupunct Meridian Stud 2018;11:83-7.

9. Lim TK, Ma Y, Berger F, Litscher G. Acupuncture and neural mechanism in the management of low back pain-an update. Medicines (Basel) 2018;5:63.

10. Charles D, Hudgins T, MacNaughton J, Newman E, Tan J, Wigger M. A systematic review of manual therapy techniques, dry cupping and dry needling in the reduction of myofascial pain and myofascial trigger points. J Bodyw Mov Ther 2019;23: 539-46.

11. Al-Bedah AMN, Elsubai IS, Qureshi NA, Aboushanab TS, Ali GIM, El-Olemy AT, et al. The medical perspective of cupping therapy: effects and mechanisms of action. J Tradit Complement Med 2018;9:90-7.

12. Cramer H, Klose P, Teut M, Rotter G, Ortiz M, Anheyer D, et al. Cupping for patients with chronic pain: a systematic review and meta-analysis. J Pain 2020;21:943-56.
13. Al-Bedah AM, Aboushanab TS, Alqaed MS, Qureshi NA, Suhaibani I, Ibrahim G, et al. Classification of cupping therapy: a tool for modernization and standardization. J Complement Altern Med Res 2016;1:27222.

14. Wang J, Wang D, Zhao W, Wang Y, Pei H, Shang Y, et al. Effect of cupping therapy in the treatment of low back pain among nurses in China. J Altern Complement Integr Med 2020;6:92.

15. Volpato MP, Breda ICA, de Carvalho RC, de Castro Moura C, Ferreira LL, Silva ML, et al. Single cupping thearpy session improves pain, sleep, and disability in patients with nonspecific chronic low back pain. J Acupunct Meridian Stud 2020;13:4852 .

16. Teut M, Ullmann A, Ortiz M, Rotter G, Binting S, Cree M, et al. Pulsatile dry cupping in chronic low back pain - a randomized three-armed controlled clinical trial. BMC Complement Altern Med 2018;18:115.

17. Markowski A, Sanford S, Pikowski J, Fauvell D, Cimino D, Caplan S. A pilot study analyzing the effects of Chinese cupping as an adjunct treatment for patients with subacute low back pain on relieving pain, improving range of motion, and improving function. J Altern Complement Med 2014;20:113-7.

18. Yazdanpanahi Z, Ghaemmaghami M, Akbarzadeh M, Zare $\mathrm{N}$, Azisi A. Comparison of the effects of dry cupping and acupressure at acupuncture point (BL23) on the women with postpartum low back pain (PLBP) based on short form McGill Pain Questionnaires in Iran: a randomized controlled trial. J Family Reprod Health 2017;11:82-9.

19. AlBedah A, Khalil M, Elolemy A, Hussein AA, AlQaed M, Al Mudaiheem A, et al. The use of wet cupping for persistent nonspecific low back pain: randomized controlled clinical trial. J Altern Complement Med 2015;21:504-8.

20. Kim JI, Kim TH, Lee MS, Kang JW, Kim KH, Choi JY, et al. Evaluation of wet-cupping therapy for persistent non-specific low back pain: a randomised, waiting-list controlled, open-label, parallel-group pilot trial. Trials 2011;12:146.

21. Comachio J, Oliveira Magalhães M, Nogueira Burke T, Vidal Ramos LA, Peixoto Leão Almeida G, Silva AP, et al. Efficacy of acupuncture and electroacupuncture in patients with nonspecific low back pain: study protocol for a randomized controlled trial. Trials 2015;16:469.

22. Martins ES, Tavares TMCL, Lessa PRA, Aquino PS, Castro RCMB, Pinheiro AKB. Acupuncture treatment: multidimensional assessment of low back pain in pregnant women. Rev Esc Enferm USP 2018;52:e03323.

23. Associação Brasileira de Medicina Física e Reabilitação. Chronic nonspecific low back pain: rehabilitation. Rev Assoc Med Bras (1992) 2013;59:536-53.

24. Tagliaferri SD, Miller CT, Owen PJ, Mitchell UH, Brisby H, Fitzgibbon B, et al. Domains of chronic low back pain and assessing treatment effectiveness: a clinical perspective. Pain Pract 2020;20:211-25. 
25. Hartvigsen J, Hancock MJ, Kongsted A, Louw Q, Ferreira ML, Genevay $S$, et al. What low back pain is and why we need to pay attention. Lancet 2018;391:2356-67.

26. Perissinotti DMN, Portnoi AG. Psychobehavioral and psychosocial aspects of neuropathic pain patients. Rev Dor 2016; 17(Suppl 1):S79-84.

27. de Sousa Mata M, da Costa FA, de Souza TO, de Sousa Mata ÁN, Pontes JF. [Pain and functionality in primary health care]. Cien Saude Colet 2011;16:221-30. Portuguese.

28. Jesus-Moraleida FR, Pereira LSM, Vasconcelos CM, Ferreira $\mathrm{PH}$. Multidimensional features of pain in patients with chronic neck pain. Fisioter Mov 2017;30:569-77.

29. Porporatti AL, Costa YM, Stuginski-Barbosa J, Bonjardim LR, Conti PCR. Acupuncture therapeutic protocols for the management of temporomandibular disorders. Rev Dor 2015;16:53-9.

30. Adams H, Thibault P, Ellis T, Moore E, Sullivan M. The relation between catastrophizing and occupational disability in individuals with major depression: concurrent and prospective associations. J Occup Rehabil 2017;27:405-12.

31. La Touche R, Pérez-Fernández M, Barrera-Marchessi I, Lópezde-Uralde-Villanueva I, Villafañe JH, Prieto-Aldana M, et al. Psychological and physical factors related to disability in chronic low back pain. J Back Musculoskelet Rehabil 2019;32:603-11.

32. Garbi Mde O, Hortense P, Gomez RR, da Silva Tde C, Castanho AC, Sousa FA. Pain intensity, disability and depression in individuals with chronic back pain. Rev Lat Am Enfermagem 2014;22:569-75.

33. Schulz KF, Altman DG, Moher D. CONSORT 2010 statement: updated guidelines for reporting parallel group randomized trials. Ann Intern Med 2010;152:726-32.

34. Childs JD, Cleland JA, Elliott JM, Teyhen DS, Wainner RS, Whitman JM, et al. Neck pain: clinical practice guidelines linked to the International Classification of Functioning, Disability, and Health from the Orthopedic Section of the American Physical Therapy Association. J Orthop Sports Phys Ther 2008;38:A1-34.

35. Vaughn AR, Clark AK, Sivamani RK, Shi VY. Natural oils for skin-barrier repair: ancient compounds now backed by modern science. Am J Clin Dermatol 2018;19:103-17.

36. Yakamura Y, Yakamura ML. [Guia de Acupuntura]. Barueri: Manole; 2015. Portuguese.

37. Silva HJA, Saragiotto BT, Silva RS, Lins CAA, de Souza MC. Dry cupping in the treatment of individuals with non-specific chronic low back pain: a protocol for a placebo-controlled, randomised, double-blind study. BMJ Open 2019;9:e032416.

38. Kim TH, Kim KH, Choi JY, Lee MS. Adverse events related to cupping therapy in studies conducted in Korea: a systematic review. Eur J Integr Med 2014;6:434-40.

39. Campbell WI, Lewis S. Visual analogue measurement of pain. Ulster Med J 1990;59:149-54.

40. Vigatto R, Alexandre NM, Correa Filho HR. Development of a
Brazilian Portuguese version of the Oswestry Disability Index: cross-cultural adaptation, reliability, and validity. Spine (Phila Pa 1976) 2007;32:481-6.

41. Pilz B, Vasconcelos RA, Marcondes FB, Lodovichi SS, Mello W, Grossi DB. The Brazilian version of STarT Back Screening Tool translation, cross-cultural adaptation and reliability. Braz J Phys Ther 2014;18:453-61.

42. Espírito-Santo H, Daniel FB. [Calculating and reporting effect sizes on scientific papers (1): $\mathrm{p}<0.05$ limitations in the analysis of mean differences of two groups]. Rev Port Investig Comport Soc 2015;1:3-16. Portuguese.

43. Ostelo RW, de Vet HC. Clinically important outcomes in low back pain. Best Pract Res Clin Rheumatol 2005;19:593-607.

44. Beltrán Molano ML, Pinilla Bonilla LB, Beltrán Dussan EH, Vásquez Londoño CA. Anatomo-functional correlation between head zones and acupuncture channels and points: a comparative analysis from the perspective of neural therapy. Evid Based Complement Alternat Med 2014;2014:836392.

45. Longhurst JC. Defining meridians: a modern basis of understanding. J Acupunct Meridian Stud 2010;3:67-74.

46. Langevin HM, Yandow JA. Relationship of acupuncture points and meridians to connective tissue planes. Anat Rec 2002;269: 257-65.

47. Cho YJ, Song YK, Cha YY, Shin BC, Shin IH, Park HJ, et al. Acupuncture for chronic low back pain: a multicenter, randomized, patient-assessor blind, sham-controlled clinical trial. Spine (Phila Pa 1976) 2013;38:549-57.

48. Shipton EA. Physical therapy approaches in the treatment of low back pain. Pain Ther 2018;7:127-37.

49. Øiestad BE, Hilde G, Tveter AT, Peat GG, Thomas MJ, Dunn $\mathrm{KM}$, et al. Risk factors for episodes of back pain in emerging adults. A systematic review. Eur J Pain 2020;24:19-38.

50. Kikuchi S. The recent trend in diagnosis and treatment of chronic low back pain. Spine Surg Relat Res 2017;1:1-6.

51. Matsudaira K, Kawaguchi M, Isomura T, Inuzuka K, Koga T, Miyoshi K, et al. Assessment of psychosocial risk factors for the development of non-specific chronic disabling low back pain in Japanese workers-findings from the Japan Epidemiological Research of Occupation-related Back Pain (JOB) study. Ind Health 2015;53:368-77.

52. Ikemoto T, Miki K, Matsubara T, Wakao N. Psychological treatment strategy for chronic low back pain. Spine Surg Relat Res 2019;3:199-206.

53. Pach D, Yang-Strobel X, Lüdtke R, Roll S, Icke K, Brinkhaus $\mathrm{B}$, et al. Standardized versus individualized acupuncture for chronic low back pain: a randomized controlled trial. Evid Based Complement Alternat Med 2013;2013:125937.

54. Shin JY, Ku B, Kim JU, Lee YJ, Kang JH, Heo H, et al. Shortterm effect of laser acupuncture on lower back pain: a randomized, placebo-controlled, double-blind trial. Evid Based Complement Alternat Med 2015;2015:808425. 
55. Song CY, Lin SF, Huang CY, Wu HC, Chen CH, Hsieh CL. Validation of the Brief Pain Inventory in patients with low back pain. Spine (Phila Pa 1976) 2016;41:E937-42.

56. Akbarzadeh M, Ghaemmaghami M, Yazdanpanahi Z, Zare N, Azizi A, Mohagheghzadeh A. The effect dry cupping therapy at acupoint BL23 on the intensity of postpartum low back pain in primiparous women based on two types of questionnaires, 2012; a randomized clinical trial. Int J Community Based Nurs Midwifery 2014;2:112-20.

57. Feitosa AA, Amaro Junior E, Sanches LG, Borba EF, Jorge LL, Halpern ASR. Chronic low back pain and sick-leave: a functional magnetic resonance study. Adv Rheumatol 2020;60:
46.

58. Vibe Fersum K, O'Sullivan P, Skouen JS, Smith A, Kvåle A. Efficacy of classification-based cognitive functional therapy in patients with non-specific chronic low back pain: a randomized controlled trial. Eur J Pain 2013;17:916-28.

59. Rozenfeld E, Kalichman L. New is the well-forgotten old: the use of dry cupping in musculoskeletal medicine. J Bodyw Mov Ther 2016;20:173-8.

60. Moura CC, Chaves ÉCL, Cardoso ACLR, Nogueira DA, Corrêa HP, Chianca TCM. Cupping therapy and chronic back pain: systematic review and meta-analysis. Rev Lat Am Enfermagem 2018;26:e3094. 